\title{
AN EMISSION-LINE GALAXY SURVEY WITH THE UK SCHMIDT TELESCOPE
}

M. Kalafi, University of Tabriz, IRAN

A. Savage, A.R. Good and R.D. Cannon, Royal Observatory, Edinburgh, U.K.

M.G. Yates, University of Edinburgh, U.K.

\section{INTRODUCTION}

The use of objective prisms in conjunction with the large area coverage afforded by Schmidt telescopes provides a very powerful means of detecting large numbers of emission-line galaxies, and allows one to study their large scale distribution. An important question that has yet to be fully addressed is the relationship between the number-magnitude distributions of the normal field galaxy and emission-line galaxy populations. A comparison such as this would effectively probe the evolution with time of these active objects. For example, study of the distant $(z=0.458)$ cluster of galaxies associated with 3C 295 (Dressler \& Gunn 1983) indicates that emission-line objects may have been far more numerous in the past than at present. As a preliminary investigation in advance of a larger project, we report here on a search for emission-line galaxies in four United Kingdom $1.2 \mathrm{~m}$ Schmidt Telescope (UKST) objective prism fields.

\section{THE SURVEY}

The choice of emulsion and spectrum dispersion best suited to emission-line object surveys is largely dependent on the type of objects it is proposed to detect and the limiting magnitude desired. The strongest emission-lines seen in galaxy spectra are [O II] $\lambda 3727, \mathrm{H} \beta \lambda 4861$, [O III] $\lambda \lambda 4959,5007$, and the blend of $\mathrm{H} \alpha \lambda 6563$ and [N II] $\lambda \lambda 6548,6584$. The prominent [O II], $\mathrm{H} \beta$ and [O III] blend are easily seen on the blue sensitive IIIa-J emulsion while the $\mathrm{H} \alpha$ and [N II], which are blended at objective prism dispersions are best seen on IIIa-F emulsions. We have thus used the IIIa-J emulsion for our survey, using IIIa-F plates only to confirm the emission-line nature of the candidate objects. Four prism dispersions are available with the UKST: $2400,1200,800 \& 600 \AA \mathrm{mm}^{-1}$ (measured at about $4300 \AA$ ) (Savage et al. 1985). We have found that the optimum for this work is $1200 \AA \mathrm{mm}^{-1}$. The combination of IIIa-J emulsion with this dispersion, without spectrum widening has a limiting magnitude $\mathrm{B}=$ 18.0. At the lowest dispersion $\left(2400 \AA \mathrm{mm}^{-1}\right)$ it is not possible to detect weak-lined objects due to poor resolution, and $\mathrm{H} \beta$ becomes blended with [O III]. The higher dispersions (e.g. $800 \AA \mathrm{mm}^{-1}$ ) have a brighter continuum limiting magnitude. Strong-lined objects can also be detected at or below the 
continuum limiting magnitude at $1200 \AA \mathrm{mm}^{-1}$. A visual search for all emission-line galaxies was made in three ESO/SERC fields, numbers $197\left(02^{\mathrm{h}}\right.$ $\left.00^{\mathrm{m}},-50^{\circ}\right), 345\left(22^{\mathrm{h}} 30^{\mathrm{m}},-40^{\circ}\right), 718\left(13^{\mathrm{h}} 00^{\mathrm{m}},-10^{\circ}\right)$ and one non-standard field $\left(22^{\mathrm{h}} 03^{\mathrm{m}},-18^{\circ} 55^{\mathrm{m}}\right)$. Each field has an area of 40 square degrees. The emission-line galaxies found on the prism plates were then classified using direct plates, into one of the following four categories:

(a) Bright galaxies with prominent HII regions.

(b) Bright galaxies with nuclear emission.

(c) Bright galaxies where the source of emission is ambiguous.

(d) Compact galaxies.

Figure 1. shows direct images of typical examples of each of these four classes, together with their corresponding prism spectra. A B magnitude for each galaxy was estimated from direct plates, as defined photographically by the IIIa-J emulsion cutoff and a GG 395 filter. This defines an effective wavelength range of $3950-5450 \AA$. At the continuum limiting magnitude $(B=18.0)$ and for spectra with a dispersion of $1200 \AA \mathrm{mm}^{-1}$ we expect to detect all objects with emission-lines of an equivalent width greater than $6 \AA$ at [O II] $\lambda 3727$. An attempt was made to compare the emission-line galaxy surveys of Fairall (1984) and MacAlpine and Lewis (1978) with our galaxy identifications. The only common area of sky was part of the Fairall survey and we identified none of his emission-line objects.

\section{RESULTS}

A catalogue of the emission-line galaxies found is currently in preparation. For three of the fields surveyed the mean density of emission-line galaxies (to $B=$ 18 ) is 0.3 per square degree but in field 718 the surface density is 1 per square degree, these numbers, down to $B \sim 15.5$, being roughly proportional to the total number of galaxies in each field. This compares well with the number densities for UV galaxy searches by Markarian et al. (1979) and Takase (1980). Analysis of field 718 indicates that the most numerous component of the emission-line galaxy population consists of compact galaxies (about 40\%). Galaxies with separate HII regions comprise about $30 \%$, those with nuclear emission only about $20 \%$ of the population, and those ambiguously identified about $10 \%$. Small number statistics prevent a similar analysis in the other 3 fields.

\section{REFERENCES}

Dressler, A. \& Gunn, J.E., 1983. Astrophys. J., 270, 7.

Fairall, A.P., 1984. Mon. Not. R. astr. Soc., 210, 69.

MacAlpine, G.M. \& Lewis, G.W., 1978. Astrophys. J. Suppl., 36, 587.

Markarian, B.E., Lipovetskii, V.A., \& Stepanian, D.A., 1979, Astrofizika, 15, 549.

Savage, A., Beard, S.M., \& Palmer, J.B., 1985. The UK Schmidt Telescope Objective Prisms, Royal Observatory Edinburgh.

Takase, B., Publ. astron. Soc. Japan, 32, 605. 

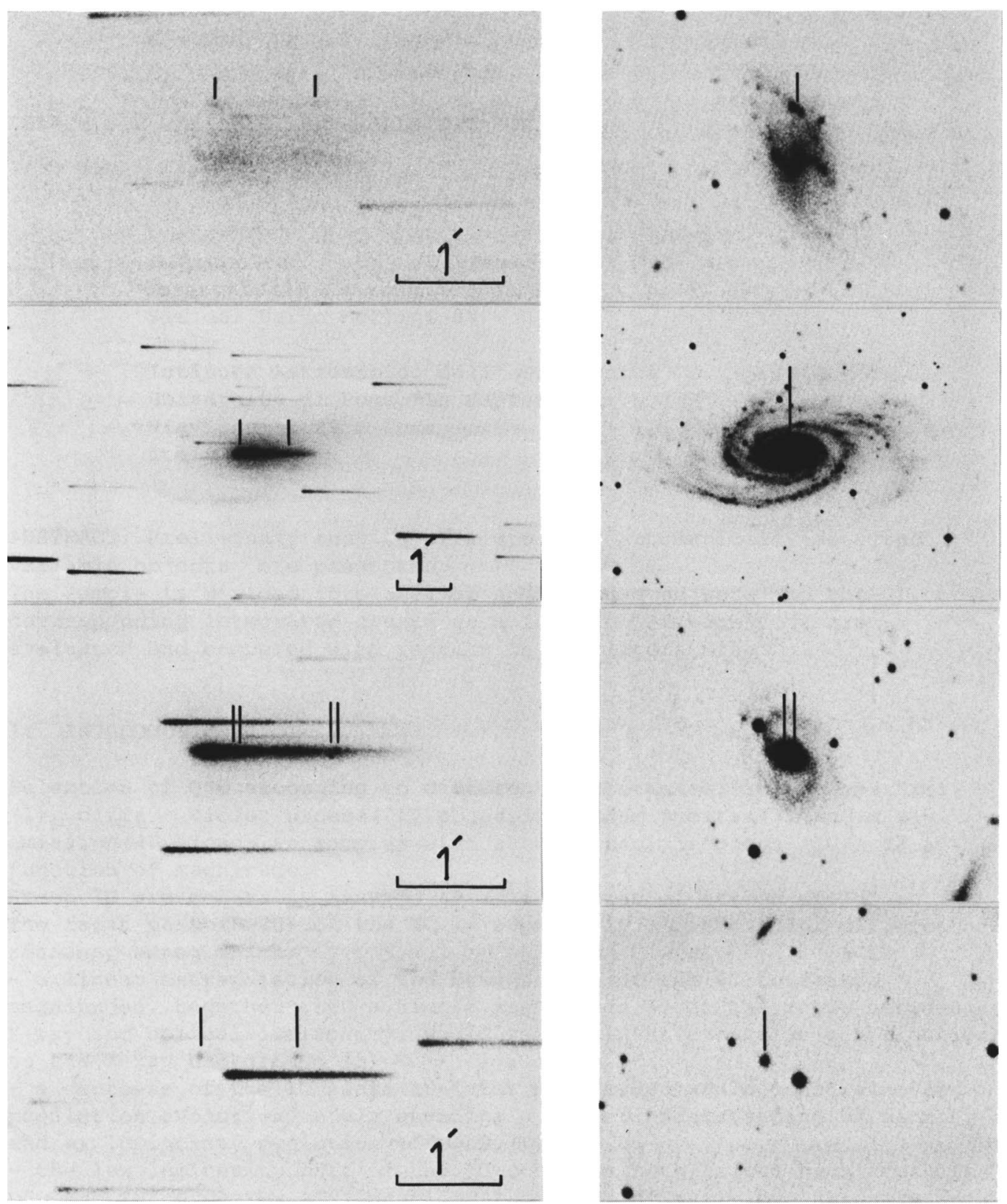

Figure 1. Representative objective prism spectra and direct images of each emission-line galaxy class.

(a) Bright galaxies with prominent HII regions.

(b) Bright galaxies with nuclear emission.

(c) Bright galaxies where the source of emission is ambiguous.

(d) Compact galaxies. 